\title{
HIPOTENSÃO CONTROLADA INDUZIDA POR HALOTANO EM COELHOS
}

\author{
HALOTHANE INDUCED CONTROLLED HYPOTENSION IN RABBITS
}

\author{
Nubia Verçosa Figueiredo ${ }^{1}$ \\ Alberto Schanaider, TCBC-RJ ${ }^{2}$ \\ Louis Barrucand ${ }^{3}$ \\ Allan Antonio da Costa ${ }^{4}$
}

\begin{abstract}
RESUMO: Objetivo: Descrever e analisar um estudo experimental com o objetivo de pesquisar o uso do halotano em diferentes concentrações, visando à obtenção de hipotensão induzida ou controlada e à análise das repercussões hemodinâmicas decorrentes do método. Método: Foram utilizados vinte e nove coelhos (Oryctolagus cuniculus), do tipo Nova Zelândia subdivididos em quatro grupos, dentre os quais havia um controle composto por cinco animais, e outros três contendo oito coelhos cada um. Os grupos I,II e III ( $\mathrm{n}=8$ cada)) foram submetidos a um período de hipotensão induzida, utilizando-se o halotano em concentrações de 1,0\%,1,5\% e 2,0\%, respectivamente. Foram avaliadas as frequiências cardíaca (FC) e respiratória (FR), a pressão arterial média (PAM) e a temperatura corporal. No sangue arterial analisaram-se os valores do potencial hidrogeniônico $(\mathrm{pH})$, da pressão parcial do oxigênio $\left(\mathrm{PaO}_{2}\right)$, da pressão parcial do gás carbônico $\left(\mathrm{PaCO}_{2}\right)$, do bicarbonato $\left(-\mathrm{HCO}_{3}\right)$, do excesso de bases $(\mathrm{BE})$, da saturação do oxigênio da hemoglobina $\left(\mathrm{Sat}_{2} \mathrm{O}_{2}\right)$. Estes parâmetros foram obtidos em três momentos: antes $(\mathrm{MAnH})$, durante $(\mathrm{MDuH})$ e após $(\mathrm{MApH})$ a hipotensão induzida. Acompanhou-se, periodicamente, os reflexos corneano e pupilar e, de forma contínua, o eletrocardiograma. Resultados: A análise estatística dos resultados evidenciou hipotensão e redução de frequência cardíaca com o uso do halotano. $\mathrm{O}$ pH dos coelhos é mais alcalino que o do homem. Não obstante haver uma tendência à acidose metabólica, esta decorreu de uma condição transitória, sem comprometer a homeostase. Conclusão: O halotano é um anestésico seguro e eficaz para promover a hipotensão induzida ou controlada, em coelhos.
\end{abstract}

Descritores: Anestesia; Halotano; Hipotensão controlada; Coelhos.

\section{INTRODUÇÃO}

A anestesia geral permite a abolição da percepção das sensações, ocorrendo perda da consciência. É obtida pela combinação de quatro elementos: hipnose, analgesia, relaxamento muscular e bloqueio dos reflexos autonômicos ${ }^{1,2}$. A hipotensão controla- da ou induzida visa a prover um campo cirúrgico exangue, melhor visualização das estruturas anatômicas e redução do tempo de cirurgia, facilitando, assim, a tarefa do cirurgião ${ }^{3,4}$. Os métodos de hipotensão controlada dependem da técnica escolhida e variam em função de modificações do tônus vascular, da pré e pós-carga, do débito cardíaco, da resistência vascular

1. Professora Adjunta do Departamento de Cirurgia da Faculdade de Medicina da UFRJ e Anestesiologista Responsável pelo Ambulatório de Anestesiologia - Avaliação Pré-anestésica do Hospital Universitário Clementino Fraga Filho (HUCFF) /UFRJ.

2. Professor Adjunto do Departamento de Cirurgia da Faculdade de Medicina da UFRJ/HUCFF.

3. Professor Titular do Departamento de Patologia da Faculdade de Medicina da UFRJ.

4. Neurocirurgião do Hospital Municipal Souza Aguiar.

Recebido em 15/05/2002

Aceito para publicação em 18/03/2003

Pesquisa desenvolvida no Serviço de Cirurgia Experimental do Departamento de Cirurgia da Faculdade de Medicina da Universidade Federal do Rio de Janeiro; Tese de doutorado do Curso de Pós-Graduação em Cirurgia Geral - Setor Anestesiologia - do Departamento de Cirurgia da Faculdade de Medicina da Universidade Federal do Rio de Janeiro. 
sistêmica e do retorno venoso ${ }^{5}$. Trata-se de procedimento que reduz a necessidade de transfusões sangüineas e suas complicações, como transmissões de doenças (síndrome de imunodeficiência adquirida, hepatite, malária, sífilis e doença de Chagas), discrasias sangüíneas, reações anafiláticas, choque pirogênico e outras. Diversos fármacos e técnicas de bloqueio foram utilizados para a obtenção da hipotensão controlada. As indicações mais comuns do método ocorrem, principalmente, no âmbito de procedimentos cirúrgicos vasculares, cardíacos, otor-rinolaringológicos, buco-maxilo-faciais, ortopédicos, plásticos, endócrinos e neurológicos ${ }^{6-8}$. Atualmente, aplica-se uma combinação de fármacos aliada à monitorização rigorosa dos sinais vitais, com o intuito de induzir, de forma eficaz, a hipotensão arterial. O halotano, anestésico escolhido para este estudo, apresenta vantagens por não ser inflamável, apresentar facilidade na administração, ter um custo razoável e proporcionar condições favoráveis para a obtenção de hipotensão arterial controlada, com diferentes concentrações ${ }^{1,9,10}$. Destarte, essa pesquisa experimental foi realizada com o objetivo de consubstanciar a eficácia e a margem de segurança da hipotensão induzida pelo halotano.

\section{MÉTODO}

Foram utilizados vinte e nove coelhos (Oryctolagus cuniculus), de ambos os sexos (treze machos e dezesseis fêmeas) do tipo Nova Zelândia , com o peso médio de $2,7 \mathrm{~kg}$, média de idade de seis meses, divididos em quatro grupos, três deles compostos por oito animais cada (grupo I, II e III) e um grupo controle $(\mathrm{C})$, contendo cinco coelhos. Estes animais foram alojados em gaiolas apropriadas, mantidos à temperatura e ventilação ambientais e com ração adequada. Com a finalidade de avaliar parâmetros de normalidade do coelho (frequiências cardíaca e respiratória, gasometria arterial, temperatura corporal), foi estudado um grupo de cinco animais, o qual não foi submetido à ação de nenhum fármaco e nem a hipotensão induzida. A coleta de sangue foi efetuada na artéria auricular central.

Cada animal, dos demais grupos, após um jejum pré-operatório de doze horas, foi conduzido à sala de cirurgia experimental, pesado e colocado em gaiola de madeira para contenção. Nesta, exteriorizavase a cabeça, através de um orifício de diâmetro fixo, mantendo-se o corpo entre anteparos laterais, evitando-se assim movimentos indesejáveis. Com a ajuda de um estetoscópio, anotavam-se as freqüências respiratória e cardíaca. A indução anestésica iniciava-se com a utilização seqüencial, a intervalos de dez minutos, por via intramuscular, das seguintes substâncias: tiopental $(20 \mathrm{mg} / \mathrm{kg})$, sulfato de atropina $(0,04 \mathrm{mg} / \mathrm{kg})$ e cetamina (ketalar- $25 \mathrm{mg} / \mathrm{kg}$ ). Prosseguia-se com tricotomia da orelha na topografia da veia marginal, cuidados de antissepsia com álcool iodado, compressão suave na base da orelha, aplicação de xilol para dilatar a veia e facilitar a introdução de um escalpe $25 \mathrm{G}$ do tipo borboleta. Mantinha-se a perfusão deste vaso, através de uma solução de ringer lactato controlada por equipo de microgotas, na razão de $4 \mathrm{ml} /$ $\mathrm{kg} / \mathrm{h}$. A partir de então os coelhos eram colocados na mesa operatória (calha de Claude Bernard) e imobilizados em decúbito dorsal. Iniciava-se o processo de manutenção da anestesia com vaporizador universal com dispositivo que permite variar as concentrações dos anestésicos inalatórios (aumentando ou diminuindo). Nesta etapa, os coelhos inalavam, sob máscara e com ventilação espontânea concentrações variáveis de halotano $(1,0 \%, 1,5 \%$ e $2,0 \%)$. A ventilação foi efetuada utilizando o sistema Rees- Baraka, avalvular com duplo T, sem absorvedor de $\mathrm{CO}_{2}$ (cal sodada) e constituído por um tubo reservatório de $60 \mathrm{ml}$ de capacidade, em cujas extremidades se conectavam uma bolsa ventilatória e uma máscara. Pode-se fazer ventilação espontanea (montagem Magill) e controlada manual (montagem Rees), na dependência do local onde estava adaptado o fluxo de admissão de gases (oxigênio e halotano). O fluxo variava de 300 a 1000 $\mathrm{ml} / \mathrm{min}$.

Para a monitoração dos animais nesta fase, utilizaram-se os seguintes parâmetros:

1) Frequiência e ritmo cardíacos: após a tricotomia da parede ventral do tórax, transfixava-se a pele com agulhas de insulina (no tórax direito, esquerdo e ao nível do coração), adaptando-se três eletrodos, os quais foram conectados ao cardioscópio na derivação $D_{\text {II }}$. 2) Freqüência respiratória: foi controlada pela introdução de um estetoscópio esofagiano que permitia ainda a monitoração da frequiência cardíaca. 3) Saturação de oxigênio da hemoglobina: após a tricotomia da pata dianteira do animal, colocava-se um sensor pediátrico, tipo oxímetro de pulso, sobre um leito vascular pulsátil. Com este monitor também se obtinha a freqüência cardíaca. 4) Temperatura corporal: introduzia-se um sensor no reto, adaptado a um termômetro do tipo digital. A temperatura corporal era mantida entre $39^{\circ} \mathrm{C} \mathrm{e} 40^{\circ} \mathrm{C}$, através de um col- 
chão térmico (cinta elétrica), posicionado sob o animal. 5) Após essa monitoração inicial, realizava-se uma traqueostomia, com inserção de uma cânula sem balonete (2,5 ou 3,0 $\mathrm{mm}$ de diâmetro), adaptado ao sistema Rees-Baraka. Nesta etapa, o animal encontrava-se ainda sob ventilação espontânea. 6) Prosseguia-se com a cateterização da artéria femoral, com cânula de teflon, com guia $\mathrm{n}^{\circ} 24 \mathrm{G}$, conectando-o a um equipo de soro heparinizado, em cuja extremidade, oposta à da artéria, se conectava um aneróide. Este permitia o controle visual contínuo da pressão arterial média. Entre o canhão da cânula de teflon e o equipo do soro, interpunha-se uma torneira de três vias para possibilitar a retirada de amostras de sangue arterial. A partir deste momento, instituía-se ventilação controlada manual, até que uma hipotensão induzida, a níveis pressóricos de 35 a 40 mm Hg (normal: 70 a 80 $\mathrm{mm} \mathrm{Hg}$ ), fosse obtida. Decorridos 60 minutos, interrompia-se a administração do anestésico inalatório e observava-se a normalização dos níveis pressóricos, com o retorno da respiração espontânea e dos reflexos, corneano e pupilar. A volemia foi mantida com solução cristalóide (ringer lactato) na dose de 4ml/ $\mathrm{kg} / \mathrm{h}$, compensando as perdas volêmicas de sangue devido a cirurgia e a traqueostomia.

Quanto à hipotensão, os parâmetros de monitoração das freqüências cardíaca e respiratória, da saturação de oxigênio, da temperatura corporal, da pressão arterial média bem como das gasometrias arteriais eram observados e anotados em três momentos: 1) antes do início da hipotensão induzida com o uso de halotano e respiração espontânea (MAnH), 2) durante a hipotensão induzida com o uso do halotano e ventilação controlada manual (MDuH), 3) após a a hipotensão porém com supressão do anestésico, com respiração assistida ou espontânea e normalização dos níveis pressóricos $(\mathrm{MApH})$.

Os dados obtidos, com enfase à média, ao desvio padrão e ao erro da média foram submetidos a análise estatística de amostras independentes pela prova de Kruskal Walis e a avaliação da significância entre as médias pelo teste de Student Newman-Keuls. Resultados com probabilidade igual ou inferior a 0,05 $(\mathrm{p} \leq 0,05)$ foram tidos como significativos. Este estudo foi efetuado de acordo com os preceitos éticos estabelecidos para os animais de experimentação ${ }^{11}$.

\section{RESULTADOS}

Os cinco coelhos do grupo controle (animais sem anestesia) apresentavam os seguintes valores: pressão arterial: $98 \pm 2,4 \mathrm{~mm} \mathrm{Hg}$, frequiência cardíaca: $230 \pm 30,8$ bpm, freqüência respiratória: $52 \pm 11,5$ ipm, potencial hidrogêniônico (pH): 7,52 $\pm 0,0391$, equilíbrio ácido-base: $+4,26 \pm 2,22 \mathrm{mEq} / \mathrm{l}, \mathrm{PO}_{2}: 86,4 \pm$ $3,63 \mathrm{~mm} \mathrm{Hg}, \mathrm{P}_{\mathrm{a}} \mathrm{CO}_{2}: 33,3 \pm 2,51 \mathrm{~mm} \mathrm{Hg}$., ${ }^{-} \mathrm{HCO}_{3}: 25,5$ $\pm 2.07 \mathrm{mEq} / \mathrm{l}$, Saturação de $\mathrm{O}_{2}: 96,9 \pm 0,7 \%$.

A hipotensão arterial foi observada nas três concentrações do halotano nos $\mathrm{MDuH}(\mathrm{p}<0.001)$. Em MAnH e MApH não houve alteração da pressão arterial (Tabela 1). A freqüência cardíaca teve redução significativa nas três concentrações do halotano nos MAnH [1\% (p=0,0062); $1,5 \%(\mathrm{p}=0,0109)$ e $2 \%$ $\mathrm{p}=0,0045]$, comparando os MDuH com os MAnH e $\mathrm{MApH}$ a $1,5 \%(\mathrm{p}=0,001)$ e a $2 \%(\mathrm{p}=0,04)$ e ao relacionar os MDuH e MApH (p=0,009) (Tabela 2). Quanto à frequiência respiratória não houve alteração nos MAnH, porém comparando os momentos e as concentrações os resultados foram: a 1\% MAnH e MApH $(\mathrm{p}=0,001)$; a $1,5 \%$ o MDuH e o MAnH ( $\mathrm{p}=0.004)$; e

Tabela 1 - Pressão Arterial média (mm de Hg).

\begin{tabular}{ccccccccccc}
\hline Estatística & \multicolumn{2}{c}{$\begin{array}{c}\text { Grupo 1: } \\
\text { Halotano 1\% } \\
(8 \text { coelhos })\end{array}$} & \multicolumn{4}{c}{$\begin{array}{c}\text { Grupo 2: } \\
\text { Halotano 1,5\% } \\
(8 \text { coelhos })\end{array}$} & \multicolumn{3}{c}{$\begin{array}{c}\text { Grupo 3: } \\
\text { Halotano 2\% } \\
(8 \text { coelhos })\end{array}$} \\
\hline & Antes & Durante & Após & Antes & Durante & Após & Antes & Durante & Após \\
\hline média & 81,6 & 36,0 & 79,5 & 82,5 & 33,1 & 80,9 & 80,6 & 35,0 & 79,1 \\
$\begin{array}{c}\text { Desvio } \\
\text { padrão }\end{array}$ & 8,35 & 5,01 & 4,78 & 5,35 & 3,72 & 4,09 & 5,63 & 3,78 & 6,66 \\
$\begin{array}{c}\text { Erro da } \\
\text { média }\end{array}$ & 2,95 & 1,77 & 1,69 & 1,89 & 1,32 & 1,44 & 1,99 & 1,34 & 2,36 \\
\hline
\end{tabular}


Tabela 2 - Freqüência cardíaca (número de batimentos/min).

\begin{tabular}{ccccccc}
\hline Estatística & \multicolumn{2}{c}{$\begin{array}{c}\text { Grupo 2: } \\
\text { Halotano 1.5\% } \\
(8 \text { coelhos })\end{array}$} & \multicolumn{4}{c}{$\begin{array}{c}\text { Grupo 3: } \\
\text { Halotano 2\% } \\
(8 \text { coelhos })\end{array}$} \\
\hline & Antes & Durante & Após & Antes & Durante & Após \\
\hline média & 197,5 & 179,4 & 198,1 & 203,1 & 168,8 & 198,8 \\
Desvio padrão & 12,8 & 11,5 & 9,98 & 16,2 & 23,0 & 15,8 \\
Erro da média & 4,53 & 4,06 & 3,53 & 5,74 & 8,11 & 5,57 \\
\hline
\end{tabular}

Tabela 3 - Freqüência respiratória (número de incursões/min).

\begin{tabular}{|c|c|c|c|c|c|c|c|c|c|}
\hline \multirow[t]{2}{*}{ Estatística } & \multicolumn{3}{|c|}{$\begin{array}{c}\text { Grupo 1: } \\
\text { Halotano 1\% } \\
\text { (8 coelhos) }\end{array}$} & \multicolumn{3}{|c|}{$\begin{array}{c}\text { Grupo } 2: \\
\text { Halotano 1,5\% } \\
(8 \text { coelhos })\end{array}$} & \multicolumn{3}{|c|}{$\begin{array}{c}\text { Grupo } 3: \\
\text { Halotano 2\% } \\
\text { (8 coelhos) }\end{array}$} \\
\hline & Antes & Durante & Após & Antes & Durante & Após & Antes & Durante & Após \\
\hline média & 57,5 & 47,1 & 62 & 55,6 & 43,1 & 55,6 & 51,3 & 35,0 & 53,1 \\
\hline $\begin{array}{l}\text { Desvio } \\
\text { padrão }\end{array}$ & 2,51 & 4,19 & 4,72 & 11,5 & 10,7 & 9,43 & 11,3 & 7,07 & 9,98 \\
\hline $\begin{array}{c}\text { Erro da } \\
\text { média }\end{array}$ & 0,886 & 1,48 & 1,67 & 4,06 & 3,77 & 3,33 & 3,98 & 2,5 & 3,53 \\
\hline
\end{tabular}

Tabela 4 - Potencial hidrogeniônico $(p H)$.

\begin{tabular}{ccccccccccc}
\hline Estatística & \multicolumn{2}{c}{$\begin{array}{c}\text { Grupo 1: } \\
\text { Halotano 1\% } \\
(8 \text { coelhos })\end{array}$} & \multicolumn{4}{c}{$\begin{array}{c}\text { Grupo 2: } \\
\text { Halotano 1,5\% } \\
(8 \text { coelhos })\end{array}$} & \multicolumn{4}{c}{$\begin{array}{c}\text { Grupo 3: } \\
\text { Halotano 2\% } \\
(8 \text { coelhos })\end{array}$} \\
\hline & Antes & Durante & Após & Antes & Durante & Após & Antes & Durante & Após \\
\hline média & 7,47 & 7,50 & 7,48 & 7,48 & 7,49 & 7,47 & 7,51 & 7,52 & 7,51 \\
$\begin{array}{c}\text { Desvio } \\
\text { padrão }\end{array}$ & 0,021 & 0,013 & 0,015 & 0,01 & 0,016 & 0,01 & 0,03 & 0,029 & 0,021 \\
$\begin{array}{c}\text { Erro da } \\
\text { média }\end{array}$ & 0,007 & 0,005 & 0,005 & 0,00655 & 0,00565 & 0,00435 & 0,011 & 0,0103 & 0,0075 \\
\hline
\end{tabular}

MDuH x MApH ( $\mathrm{p}=0,026)$; a $2 \%$ o MDuH e MAnH $(\mathrm{p}=0,005)$ e MDuH x MApH ( $\mathrm{p}=0,001)$ (Tabela 3), O $\mathrm{pH}$ mais alcalino foi significativo com $1 \%$ de halotano ao comparar o MDuH x MAnH (p=0,011) e MDuH x $\mathrm{MApH}(\mathrm{p}=0,015)$ (Tabela 4). A PaO $0_{2}$ esteve sempre aumentada em todas as concentrações nos MAnH $(\mathrm{p}=0.015)$; a $1 \%$ o MDuH x MAnH $(\mathrm{p}=0,0499)$ e a $1,5 \%(\mathrm{p}=0,0069)$ (Tabela 5). A $\mathrm{PaCO}_{2}$ com halotano a $1 \%$ e $1,5 \%$ acareando o $\mathrm{MDuH}$ e o $\mathrm{MAnH}(\mathrm{p}=0.02)$ e $(\mathrm{p}=0,003)$ e com o MApH $(\mathrm{p}=0,001)$ respectivamente (Tabela 6).

$\mathrm{O}$ bicarbonato $\left(\mathrm{HCO}_{3}\right)$, o excesso de bases (BE), a saturação de oxigênio da hemoglobina $\left(\mathrm{SatO}_{2}\right)$, a temperatura corporal $\left({ }^{\circ} \mathrm{C}\right)$ e o ECG não apresentaram diferenças significativas nas três concentrações do halotano nos três momentos estudados. 
Tabela 5 - Pressão parcial de oxigênio $\left(\mathrm{P}_{a} \mathrm{O}_{2}-m m \mathrm{Hg}\right)$.

\begin{tabular}{cccccccccc}
\hline Estatística & \multicolumn{2}{c}{$\begin{array}{c}\text { Grupo 1: } \\
\text { Halotano 1\% } \\
(8 \text { coelhos })\end{array}$} & \multicolumn{3}{c}{$\begin{array}{c}\text { Grupo 2 : } \\
\text { Halotano 1,5\% } \\
(8 \text { coelhos })\end{array}$} & \multicolumn{3}{c}{$\begin{array}{c}\text { Grupo 3 : } \\
\text { Halotano 2\% } \\
(8 \text { coelhos })\end{array}$} \\
\hline & Antes & Durante & Após & Antes & Durante & Após & Antes & Durante & Após \\
\hline média & 109,2 & 115,6 & 110,5 & 101,9 & 110,4 & 105,8 & 114,0 & 119,4 & 112,5 \\
$\begin{array}{c}\text { Desvio } \\
\text { padrão }\end{array}$ & 6,11 & 4,47 & 7,05 & 7,38 & 6,59 & 7,23 & 7,58 & 5,63 & 7,82 \\
$\begin{array}{c}\text { Erro da } \\
\text { média }\end{array}$ & 2,16 & 1,58 & 2,49 & 2,61 & 2,33 & 2,55 & 2,68 & 1,99 & 2,76 \\
\hline
\end{tabular}

Tabela 6 - Pressão parcial de gás carbônico $\left(\mathrm{P}_{a} \mathrm{CO}_{2}-\mathrm{mm} \mathrm{Hg}\right)$.

\begin{tabular}{|c|c|c|c|c|c|c|c|c|c|}
\hline \multirow[t]{2}{*}{ Estatística } & \multicolumn{3}{|c|}{$\begin{array}{c}\text { Grupo 1: } \\
\text { Halotano 1\% } \\
\text { (8 coelhos) }\end{array}$} & \multicolumn{3}{|c|}{$\begin{array}{c}\text { Grupo } 2: \\
\text { Halotano 1,5\% } \\
(8 \text { coelhos })\end{array}$} & \multicolumn{3}{|c|}{$\begin{array}{c}\text { Grupo } 3: \\
\text { Halotano 2\% } \\
\text { (8 coelhos) }\end{array}$} \\
\hline & Antes & Durante & Após & Antes & Durante & Após & Antes & Durante & Após \\
\hline média & 22,25 & 23,12 & 25,75 & 23,71 & 22,0 & 25,75 & 23,8 & 22,0 & 25,8 \\
\hline $\begin{array}{l}\text { Desvio } \\
\text { padrão }\end{array}$ & 1,75 & 1,45 & 0,88 & 0,7 & 1,19 & 0,86 & 0,71 & 1,20 & 0,89 \\
\hline $\begin{array}{l}\text { Erro da } \\
\text { média }\end{array}$ & 0,62 & 0,78 & 0,25 & 0,42 & 0,31 & 0,31 & 0,25 & 0,42 & 0,31 \\
\hline
\end{tabular}

\section{DISCUSSÃO}

O coelho foi o animal de experimentação escolhido, pelo fato de ser largamente utilizado em procedimentos cirúrgicos biomédicos, porque seus parâmetros hemodinâmicos são bem conhecidos. A contenção em gaiolas apropriadas viabiliza os acessos vasculares e permite a administração de substâncias por via parenteral ${ }^{12}$.

O tiopental, a atropina e a cetamina, administrados em doses preconizadas pela literatura, para a indução da anestesia, não interferiram com as frequiências cardíaca e respiratória dos coelhos, porquanto ambas permaneceram dentro dos limites de normalidade. A cetamina provoca o aumento da secreção salivar e por este motivo, foi adotado o uso de atropina, precedendo a cetamina. $O$ coelho tem particularidades anatômicas específicas que dificultam a intubação orotraqueal. Apresenta pequena abertura de boca, orofaringe estreira, língua larga e protusa na sua parte posterior, laringe pequena, oblíqua e anteriorizada, além de dentes incisivos proeminentes ${ }^{12}$. Deste modo, optamos pela traqueostomia. O tubo escolhido deve ter entre 2,5 a 3,5 mm de diâmetro interno, de acordo com a idade do animal. Não está indicado o uso do tubo traqueal com balonete, devido à pressão alta que este poderá exercer sobre o epitélio traqueal, ocasionando, às vezes, necrose dos tecidos. A volemia foi mantida com solução cristalóide (ringer lactato) na dose de $4 \mathrm{~mL} / \mathrm{kg} / \mathrm{h}$.

A manutenção da anestesia deu-se através do controle de admissão de gases (oxigênio) pelo fluxômetro associado ao vaporizador que permitia concentrações variáveis do halotano. O sistema de ventilação do tipo duplo T de Baraka, utilizado nessa pesquisa, revela ótima adequação para animais de pequeno porte, em face da pequena resistência do fluxo gasoso. Em caso de excesso de fluxo este escapará livremente pela saída próxima ao balão reservatório. Permite ainda as montagens Magill (ventila- 
ção espontânea) e Rees (ventilação controlada), dependendo da variação do local de entrada do fluxo de admissão de gases ao sistema. O cálculo do volume minuto $\left(\mathrm{V}=150-200 \mathrm{~mL} \cdot \mathrm{kg}^{-1} \mathrm{~min}^{-1}\right)$ pode ser realizado pela consulta a nomogramas que correlacionam idade, superfície corporal e o peso. Nos coelhos do nosso estudo, o fluxo de admissão de gases variou entre 300 a $1000 \mathrm{~mL}(0,3$ a $1 \mathrm{~L})$. O ideal seria utilizar o sistema com absorvedor de $\mathrm{CO}_{2}$ que aproveita os gases expirados e permite a utilização de baixos fluxos de admissão de gases, umidifica e aquece os vapores anestésicos evitando a perda de calor pela eliminação do vapor d'água para a atmosfera, além de ser mais econômico. Não o utilizamos porque não dispunhamos de aparelho com este dispositivo.

A anestesia inalatória promove uma indução rápida e permite uma metabolização e eliminação precoces dos fármacos, facilitando o controle dos planos anestésicos. $\mathrm{O}$ halotano $\left(\mathrm{CF}_{2} \mathrm{CHClBr}\right)$, fármaco escolhido para esta pesquisa, é um anestésico inalatório não inflamável e de fácil administração. O seu coeficiente de partição sangue/gás é de 2.3 , sendo portanto, moderadamente solúvel no sangue. A concentração alveolar mínima (CAM) à pressão de uma atmosfera, previne a resposta motora a estímulos nociceptivos em $50 \%$ dos pacientes ou animais. No homem a CAM do halotano é 0,7 e no coelho, situase em $1,39 \pm 0,23{ }^{12}$. Produz hipotensão arterial por deprimir o miocárdio, diminuir o débito cardíaco e o tônus do sistema nervoso simpático, além de promover vasodilatação periférica. Os efeitos adversos são a perda da auto-regulação cerebral, a redução na pressão de perfusão cerebral e o aumento na pressão intracraniana. Quanto ao inotropismo, constatou-se uma diminuição do número de batimentos, decorrente de uma inibição dos barorreceptores. Assim, não há o aparecimento de taquicardia em resposta à hipotensão. Também atribui-se ao halotano uma ação vasodilatadora $^{1,6}$. Contudo, a principal causa da hipotensão é a diminuição do débito cardíaco ${ }^{1,2,9}$. Reduz, ademais, a complacência venosa e, nos pacientes com insuficiência cardíaca, diminui a resistência vascular sistêmica. Nesta pesquisa, observamos uma diminuição da frequiência cardíaca nos três grupos, em concomitância à redução da pressão arterial. O halotano pode causar depressão do sistema respiratório e do sistema nervoso central ${ }^{10}$. Os resultados obtidos atestaram uma diminuição da freqüência respiratória em todas as concentrações utilizadas no pe- ríodo de hipotensão. Atribui-se este achado à necessidade de manter-se os animais com pressão arterial entre 30 a $40 \mathrm{mmHg}$ e sob respiração controlada manual. Quando da interrupção da inalação do anestésico e da ventilação controlada, obviamente houve elevação da frequiência respiratória. Aduz-se, ainda, como explicação ao fato, a presença de estímulo doloroso e a superficialização da anestesia.

Algumas das significâncias resultantes da comparação entre dados experimentais relacionamse mais com o procedimento correto dos ensaios ao diminuir os desvios das médias do que causadas por alterações de grande significância clínica. É o que se pode avaliar a seguir.

No que diz respeito à análise dos gases arteriais, o coelho em condições fisiológicas tem um pH sangüíneo mais alcalino ${ }^{13-15}$ que o homem (de 7,4 a 7,58). As discretas variações do $\mathrm{pH}$, sempre dentro da faixa da normalidade, podem ser atribuídas ao sistema de ventilação utilizado. $\mathrm{A}^{\mathrm{PaO}_{2}}$ encontrada nos diversos grupos estava acima do normal em decorrência da ventilação controlada e do fluxo de admissão de gases (100\% de oxigênio) variáveis de acordo com o volume minuto. Observações na literatura ${ }^{16,17}$ comprovam a mesma alteração supra citada (média $=114 \%$ e normal $=97$ a $100 \%$ ). $\mathrm{A} \mathrm{PaCO}_{2}$ esteve sempre abaixo dos volumes normais, em todos os grupos (média $=23,07 \% \mathrm{e}$ normal $=38$ a $42 \%$ ). A $\mathrm{PaCO}_{2}$, sob uma óptica clínica, variava muito pouco nas concentrações de 1,0 e $1,5 \%$, observando-se a aglutinação dos menores valores na concentração de $2 \%$. Este resultado é conseqüente à hiperventilação realizada durante o procedimento e à não utilização da cal sodada que junto ao fluxo de admissão de gases permite a reabsorção do $\mathrm{CO}_{2}$ expirado. A observação de níveis de $-\mathrm{HCO}_{3}$ reduzidos, deve-se a um mecanismo de compensação renal. Neste, o ácido lático ao transformar-se em lactato de sódio, extrai do bicarbonato o ionte sódio, formando o ácido carbônico. Cabe ressaltar que mesmo com uma tendência à acidose metabólica, o coelho tem $\mathrm{pH}$ alcalino e portanto são mínimas as variações dos mecanismos homeostáticos ácidobases. Quanto ao BE (excesso de bases), resultaram concentrações que provam uma acidose metabólica. Tal fato é conseqüência de diversos fatores: jejum pré-operatório, manipulação do animal à semelhança do observado na prática clínica, ventilação alveolar, hipóxia tecidual por hipotensão arterial, e mecanismos compensatórios renais. Não foi utili- 
zado o bicarbonato de sódio para reverter a acidose metabólica, porque tratava-se de um fenômeno transitório, sem repercussões clínicas. A $\mathrm{SatO}_{2}$ da hemoglobina manteve-se dentro dos limites de normalidade. Portanto, o efeito do halotano em diferentes concentrações não interferiu nos resultados encontrados, tanto nas gasometrias arteriais como, no oxímetro de pulso.
Não foram registradas arritmias cardíacas durante o experimento.

A análise dos resultados deste estudo comprova que a hipotensão induzida por halotano é uma técnica segura em coelhos, desde que o mesmo seja utilizado em concentrações adequadas, com monitorização rigorosa, de forma contínua, sob o controle de pessoal médico especializado.

\begin{abstract}
Background: The authors describe haemodynamic changes secondary to controlled and induced hypotension during anesthesia with different halothane concentrations. Methods: Twenty-nine New Zealand white rabbits (Oryctolagus cuniculus) were divided into four groups. A control group, composed by five animals, was compared to groups I, II, and III, with eight rabbits each one $(n=8)$, that underwent anesthesia with halothane in concentrations of 1,0\%, 1,5\% and 2,0\% respectively. Cardiac and respiratory rates, body temperature, average arterial blood pressure, $\mathrm{pH}$ values, arterial oxygen tension $\left(\mathrm{PaO}_{2}\right)$, arterial carbon dioxide tension $\left(\mathrm{PaCO}_{2}\right)$, bicarbonate concentrations ( $\left.{ }^{-} \mathrm{HCO}_{3}\right)$, base excess $(\mathrm{BE})$, and haemoglobin oxygen saturation ( $\mathrm{Sat}_{2}$ ) were studied. All parameters were registered before, during and after halothane anesthesia induced hypotension. Corneal and pupilary reflexes and cardiogram were followed continuously. Results: Halothane resulted in hypotension and reduced heart rate. Rabbits have an alcaline blood pH. The metabolic acidosis trend observed was of transient character. Halothane did not modify $\mathrm{SatO}_{2}$.Conclusion: Halothane is a safe and efficient anaesthetic to promote induced or controlled hypotension in rabbits.
\end{abstract}

Key Words: Anesthesia; Halothane; Hypotension, controlled; Rabbits.

\section{REFERÊNCIAS}

1. Steven WC, Kingston HGG - "Inhalation Anesthesia". In Barash PG, Cullen BF, Stoelting RK. - Clinical Anesthesia. $2^{\text {nd }}$ Edition. Philadelphia. J.B. Lippincott Company, 1998, pp. 439-465.

2. Ferreira MBC, Martins ALC - "Anestésicos inalatórios”. In Manica JT - Anestesiologia. Princípios e técnicas. Porto Alegre. Artes Médicas,1994. pp.123-143.

3. Rodrigues FF. Radicais livres derivados do oxigênio na isquemia cerebral transitória. Estudo experimental. Dissertação (Doutorado em Cirurgia Geral). Rio de Janeiro. Universidade Federal do Rio de Janeiro. 1992, 80 p.

4. Barker SJ, Hyatt J - Continuous measurement of intracranial $\mathrm{pH}, \mathrm{PaCO}_{2}$ and $\mathrm{PaO}_{2}$ in operating room. Anesth Analg, 1991, 73(1):43-48.

5. Nocite JR - Hipotensão arterial induzida em cirurgia. Rev Col Bras Cir, 1980, 7(3):147-152.
6. Azar I, Thiagarajah S - The jury is still out in the case of isoflurane versus halotane in neurosurgical patients. Anesthesiology, 1984, 61(6):786-787.

7. Desmonts JM, le Honelleur J, Remond P, et al. Anaesthetic management in patients with phaeochomocytoma. A review of 102 cases. Br J Anaesth, 1977, 49(10):991-998

8. Sullivan HG, Keenan RL, Isrow L, et al. - The clinical importance of $\mathrm{PaCO}_{2}$ during intracranial aneurysm surgery: case report. J Neurosurg, 1980, 52(3):426-430.

9. Mikhail Jr GE - "Inhalational Anesthetics". In Appleton \& Lange (eds) - Clinical Anesthesiology. $3^{\text {rd }}$ Edition. New York, McGraw-Hill. 2002, pp. 127-150.

10. Morgan JR, Mikail MS, Murray MJ - "Hypotensive agents". In Appleton \& Lange (eds) - Clinical Anesthesiology. $3^{\text {rd }}$ Edition. New York. McGraw-Hill. 2002, pp. 224-232.

11. Guide for the care and use of laboratory animals. Washingto DC - National Academy Press. 1996. 
12. Flecknell PA - Anaesthesia of animals for biomedical research. Br J Anaesth, 1993, 71(6): 885-894.

13. Laird CW, Fox RR, Mitchell BP, et al. - Effect of strain and age on some hematological parameters in the rabbit. Am J Phisiol, 1970, 218(6):1613-1617.

14. Fonseca NM, Goldenberg S - Sistema circular de anestesia com absorvedor de dióxido de carbono: uso em animais de pequeno porte. Acta Cir Bras, 1995; 10(.3):144-150.

15. Field EJ - Anaesthesia in rabbits. J Anim Tech Assoc, 1957, 47-48.

16. McKinney MS, Fee JP - Cardiovascular effects of $50 \%$ nitrous oxide in older adult patients anaesthetized with isoflurane or halothane. Br J Anaesth, 1998, 80 (2):169-173.
17. Figueiredo NV. Hipotensão controlada induzida por halotano. Estudo experimental em coelhos. Dissertação (Doutorado em Cirurgia Geral) Rio de Janeiro. Universidade Federal do Rio de Janeiro, 1996, $74 \mathrm{p}$.

Endereço para correspondência:

Alberto Schanaider

Rua Eurico Cruz, 33 - apto. 603 - Jardim Botânico 22241-200 - Rio de Janeiro - RJ 